\title{
EL USO DEL DICCIONARIO EN EL AULA DE EDUCACIÓN PRIMARIA
}

The use of the dictionary at the primary education classroom

Irene Carvajal Muñoz

Sarita Marín Córdoba

Jennifer Ortega Valverde*

\begin{abstract}
RESUMEN
El diccionario, una gran herramienta a la que no se le ha dado la importancia necesaria. En primaria se asume que enseñando el orden alfabético, el estudiante ya está capacitado para utilizar el diccionario y, en secundaria, se tiene por visto. De tal manera, que en la vida cotidiana, muy pocas personas utilizan el diccionario. El diccionario facilita el aprendizaje de léxico, de gramática, de ortografía, de la variación semántica y es de gran ayuda a la hora de redactar; por este motivo, hemos desarrollado unas estrategias didácticas para aprender a usarlo y acostumbrarse a utilizarlo con mayor frecuencia. Las actividades lúdicas están diseñadas para el aula de primaria, pero con algunas modificaciones se pueden utilizar para secundaria. Palabras clave: Diccionario, estrategias didácticas, educación primaria, juegos.
\end{abstract}

\begin{abstract}
The dictionary is a very useful tool that it is not being given the necessary importance. It is supposed that students will be capable to use the dictionary just teaching the alphabetic order in elementary school; in high school this is for granted. So, very few people use the dictionary on daily bases. The dictionary helps to learn vocabulary, grammar, spelling, semantic variations, and when writing; for this reason, it has been developed didactic strategies to learn how to use it and get use to use it regularly. The ludic activities are designed to the elementary school rooms, but with some modifications they could be used in high school.
\end{abstract}

Key Words: dictionary, didactic strategies, elementary education, games.

Universidad de Costa Rica. Escuela de Formación Docente. Costa Rica

Correo electrónico: nenecarvajal@gmail.com, saritamarinc@yahoo.es, jeniortega@gmail.com.

Recepción: 9/6/2013 Aceptación: 15/11/2013. 


\section{Introducción}

El diccionario es una herramienta útil para el enriquecimiento léxico, pero su utilización en el aula escolar se ha limitado a la búsqueda de palabras cuyo significado es desconocido o el orden alfabético. En la actualidad en Costa Rica existen pocas investigaciones acerca del tema por lo que el proyecto es pionero con respecto a este contenido. En nuestro país únicamente existen estudios de Marielos Murillo, Víctor Sánchez y Gabriela Ríos; quienes se distinguen por el apoyo al programa ELEXHICÓS de la Universidad de Costa Rica.

El Ministerio de Educación Pública no plantea en sus programas el uso del diccionario en objetivos específicos como tal; sino que se encuentra inmerso en otros. El proyecto plantea como un objetivo específico la propuesta didáctica de actividades lúdicas para facilitar la incorporación del contenido en las aulas escolares.

Existen muchas habilidades que pueden ser reforzadas o estimuladas por medio del uso del diccionario, pero debido al desconocimiento de los mismos docentes; por falta de capacitación; u otros factores como la falta de concientización y motivación del personal se descuida el uso de esta herramienta en nuestras aulas escolares.

Se debe tener presente que incluso las personas desconocen que existen diccionarios de diversos tipos y no existe un diccionario adecuado sino que hay múltiples que nos pueden ayudar a resolver las dudas o consultas de acuerdo a las necesidades que requiramos en un momento dado.

La metodología del proyecto fue una encuesta dirigida a los docentes de tercero y sexto grado específicamente. Posteriormente se realizaron actividades lúdicas con los estudiantes para corroborar los datos que se obtuvieron mediante las encuestas.

La idea es concientizar al personal docente en formación y en ejercicio de la labor y el papel que cumplen para poder llevar estrategias a los discentes que puedan utilizarse para aprender el adecuado y divertido uso del diccionario y no dejarlo como un objetivo más del programa de estudios. Con el fin de facilitar la labor docente en cuanto al uso del diccionario en el aula y su adecuada manipulación, nos hemos propuesto crear estas estrategias, las cuales contienen diversas actividades para trabajar este contenido por medio de una metodología lúdica. Además permiten al docente sacar el máximo provecho a todas las utilidades del diccionario más allá de buscar simples significados.

Esta propuesta didáctica consiste en ocho estrategias que tienen la finalidad de facilitar el trabajo docente para la práctica y la utilización del diccionario en el aula, para que por medio de ellas, los estudiantes aprendan qué es un diccionario, cómo es y las diferentes utilidades que esta herramienta tiene en su proceso educativo.

\section{Creación de poemas}

Objetivo: Conocer y utilizar diferentes diccionarios electrónicos y sus usos en actividades cotidianas.

$\underline{\text { Recursos: }}$ computadoras con servicio de Internet Nivel: segundo ciclo

Tiempo probable: dos lecciones.

Con la ayuda del diccionario Clave en internet: www.clave.librosvivos.net, se entregará a los estudiante un dibujo o una temática en especial, con la que deberán crear un poema, utilizando el diccionario pueden buscar palabras que "terminan con..." para crear la rima del poema. Estos poemas serán luego declamados ante la clase.

\section{Rally de palabras}

Objetivo: Realizar una búsqueda rápida y ágil en el diccionario de palabras relacionadas con un tema de estudio específico.

Recursos: fichas de colores con las palabras.

Nivel: segundo ciclo.

Tiempo probable: dos lecciones. 
Consistirá en dividir al grupo en varios subgrupos, con una cantidad similar de miembros. A cada uno se le asignará un color, el cual corresponderá a las tarjetas con las palabras de ese equipo. Se colocarán palabras relacionadas a uno de los temas en estudio, en varios lugares del aula (o de la escuela), se entregará a los estudiantes la primera palabra, la cual deberán buscar en el diccionario. Cada vez que encuentren el significado de una palabra deberán anotarlo en la ficha de esa pista, luego buscar otra tarjeta dentro del aula e ir investigando su significado, así sucesivamente hasta completar todas las pistas. Ganará el equipo que complete todas las fichas y descubra el tema de estudio.

\section{Juego de palabras}

Objetivo: Conocer el diccionario y buscar en él palabras relacionadas a diferentes temáticas.

Recursos: tablero creado por el docente, fichas para cada estudiante, dado, tarjetas de preguntas y comodines.

Nivel: primer ciclo.

Tiempo probable: dos lecciones.

El docente creará un tablero, el cual estará formado por casillas numeradas y espacios destinados a preguntas y comodines, cada subgrupo tendrá su propio tablero e iniciarán el juego lanzando un dado y avanzando la cantidad de espacio que indique el mismo, si algún estudiante cae en la casilla de preguntas deberá buscar la información que se le solicite en el diccionario, a su vez si cae en la casilla de comodines recibirá alguna bonificación, como avanzar espacios.

\section{Stop}

Objetivo: Conocer el diccionario y buscar en él palabras relacionadas a diferentes temáticas.

Recursos: tablero creado por el docente, fichas para cada estudiante, dado, tarjetas de preguntas y comodines.
Nivel: primer ciclo.

Tiempo probable: dos lecciones.

El docente creará un tablero, el cual estará formado por casillas numeradas y espacios destinados a preguntas y comodines, cada subgrupo tendrá su propio tablero e iniciarán el juego lanzando un dado y avanzando la cantidad de espacio que indique el mismo, si algún estudiante cae en la casilla de preguntas deberá buscar la información que se le solicite en el diccionario, a su vez si cae en la casilla de comodines recibirá alguna bonificación, como avanzar espacios.

\section{Parejas de palabras}

Objetivo: Conocer el diccionario y sus partes para localizar palabras con rapidez.

Recursos: tarjetas elaboradas por el docente.

Nivel: primer y segundo ciclo.

Tiempo probable: dos lecciones.

Consiste en resolver ejercicios en los cuales una palabra cumple diversos significados según la idea que plantea la oración y también se ponen en práctica parónimos y homónimos.

Se tienen tarjetas con oraciones incompletas y tarjetas con palabras que completan las oraciones de manera correcta. Los estudiantes deben encontrar la palabra que completa correctamente la oración según el sentido lógico de la misma.

Una variación de esta estrategia es que se trabaje con los significados de cada una de las palabras homónimas a manera de juego de memoria.

\section{Sopa de letras}

Objetivo: Realizar una búsqueda ágil en el diccionario.

Recursos: sopa de letras elaborada por el docente.

Nivel: segundo ciclo.

Tiempo probable: dos lecciones.

El docente entregará a los estudiantes una ficha que contenga un crucigrama y las definiciones de cada una de las palabras contenidas en 
el mismo. Estas palabras deberán tener un tema en común. Los educandos, con ayuda del diccionario, buscarán las definiciones para descubrir los vocablos, los cuales encerrarán en el crucigrama.

\section{¿Quién quiere ser millonario?}

Objetivo: Promover el uso del diccionario de manera lúdica para que el estudiante conozca y utilice en sus actividades cotidianas.

Recursos: preguntas elaboradas por el docente.

Nivel: segundo ciclo.

Tiempo probable: dos lecciones.

Se dividirá en subgrupos tratando de que cada uno tenga la misma cantidad de estudiantes, cada uno debe elegir un representante que se sentará "en la silla caliente" para responder a la pregunta correspondiente para fomentar la participación de todos los integrantes se puede elegir un participante diferente para la solución de cada reto.

El docente debe definir la cantidad de tiempo para responder tomando en cuenta las habilidades adquiridas respecto al uso del diccionario; además es necesario tener un cronómetro, y una señal de aviso para el momento del inicio y conclusión del tiempo de reacción. El docente lee la pregunta y brinda las tres posibles respuestas, cuando se da la señal de conclusión del tiempo, cada representante de los subgrupos que se encuentra en la silla caliente, externa la respuesta y se determina si esta es correcta o errónea, y se registra el número de aciertos que va teniendo cada subgrupo y se registra el número de aciertos que va teniendo cada subgrupo.

Dentro de la dinámica de la estrategia, en caso de que los participantes enfrenten alguna dificultad al momento de resolver el reto, pueden hacer uso de siguientes comodines, el comodín aplica su uso solamente una vez durante todo el desarrollo de la actividad:

- $\quad$ Solicitud de apoyo de un compañero: el representante que se encuentra en la "silla caliente", puede elegir el apoyo de un compañero que sea parte de su subgrupo.

- Derecho a obtener una pista: se puede solicitar al docente una pista que apoye a la resolución del acertijo.

- Apoyo del subgrupo al que pertenece: se tiene la posibilidad de preguntar al subgrupo la duda que se tenga en el momento.

\section{Juego de sillas}

Objetivo: Agilizar la búsqueda de palabras en el diccionario por parte de los discentes.

Recursos: sillas, tarjetas elaboradas por el docente.

Nivel: a partir de tercer grado.

Tiempo probable: dos lecciones.

Consiste en que se eligen 6 estudiantes y se colocan 5 sillas. Al escuchar la música los estudiantes caminan (con las manos hacia atrás, con la finalidad de que no se toquen los asientos) alrededor de las sillas, la docente en un determinado momento debe detener la música, a su vez todos los alumnos buscan una silla y proceden a sentarse. La persona que no obtuvo asiento debe elegir una tarjeta de la pizarra que indica un término que deberá buscar en el diccionario. El tiempo máximo para buscar el significado es 40 segundos, de lo contrario debe abandonar el juego. Las tarjetas se pueden realizar de acuerdo al tema de estudio. Las tarjetas se pueden realizar de acuerdo al tema de estudio.

\section{Conclusiones}

Se vuelve imperioso concientizar a los profesores de primaria para que el uso del diccionario se dé desde el primer grado, con actividades acordes para el nivel, potenciando un proceso gradual en cuanto a la complejidad que se incorpora grado a grado en relación a las 
habilidades necesarias para un uso correcto del diccionario.

El diccionario debe ser un objetivo y no un contenido indirecto por trabajar dentro de los planes de estudio. Incluso se hace notar en estos, en el rubro de aprendizajes por evaluar $\mathrm{y}$ objetivos que la principal idea acerca del aprendizaje del diccionario radica en aprender el orden alfabético.

El diccionario debe ser considerado como medio para formar en autonomía como un proceso de "autoaprendizaje", habilidad necesaria y de gran provecho en la vida cotidiana, para la estimulación del interés en la búsqueda y construcción del propio aprendizaje, siendo este significativo. "crear, pensar y entender". Entre más sepa un estudiante el uso correcto del diccionario, más independencia tendrá en su aprendizaje. El diccionario le dará competencia léxica, por lo tanto, será un individuo con las cuatro habilidades lingüísticas (hablar, leer, escuchar, escribir) bien desarrolladas, en otras palabras, un estudiante que tendrá una gran ventaja en el aprendizaje.

Es necesario que los docentes tomen conciencia de la importancia de su papel en la formación de los educandos en el tema del uso del diccionario, para que este punto sea alcanzado, el profesorado debe utilizar dentro de las aulas, actividades lúdicas para que los discentes encuentren en el diccionario una herramienta útil y atractiva en el proceso enseñanza - aprendizaje.

\section{Referencias bibliográficas}

Águila Escobar, Gonzalo. (2009). Los diccionarios electrónicos del español. Madrid: Arco Libros.

Alzola, L. (2000). "Las partes introductorias en la lexicografía escolar. Análisis de sus posibilidades didácticas”. En Rusteler y Prado Aragonés (Editores). Tendencias en el diccionario como objeto de estudio lingüístico y didáctico. España: Universidad de Huelva. 259-273pp.
Arauz, Carlos. (2008). Dichos y refranes costarricenses. Frases y expresiones de nuestra habla popular. San José: C. Arauz R.

Barragan Rosssana. (2003). Proyectos de Investigación. La Paz: Fundación. Bolivia.

Cassany, Daniel. et. alii. (1997). Enseñar lengua. Barcelona: Editorial GRAÓ.

Cote, M. y Tejedor, C. (s.f.). La enseñanza de vocabulario y el uso de la lengua.

D’Alton, Cristina. (1998). Lengua y Literatura: ensayos didácticos. San José: Editorial Universidad Estatal a Distancia.

Dubois, Jean. [1979(1998)]. Diccionario de lingüística. Madrid: Alianza Editorial.

Ezquerra, A. (2003). Metodología para la enseñanza del uso del diccionario, 85-100.

Gagini, Carlos. [1918(2000)]. Diccionario de costarriqueñismos. San José: Editorial Costa Rica.

García, R. (2008). Larousse. Diccionario escolar. México: Ediciones Larousse.

García, R. (1983). Diccionario Práctico Francais / Español Español- Francés Ediciones Larousse. Primera edición. Decimosexta reimpresión. Mexico.

González, N. El uso del diccionario para aprender a aprender. Caracas, Venezuela.

Gutiérrez, J. (1994). Gramática y diccionario. Madrid: Universidad de Barcelona.

Haensch, Günther. (1997). Los diccionarios del español en el umbral del siglo XXI, 
Cap. 2 (pp. 15-43). $1^{\circ}$ edición. España: Ediciones Universidad de Salamanca.

Hernández Sampieri, Roberto. et. alii (2003). Metodología de la investigación. Colombia: McGraw - Hill.

Hernández, H. (1991). De la teoría lexicográfica al uso del diccionario. España: ASELE.

Larousse. (s.f). Larousse. Diccionario Básico. Español-inglés English - Spanish. Ediciones Larousse. Primera edición- $3^{\text {a }}$ reimpresión. México.

Larousse. (s.f). Larousse. Diccionario Básico. Español-inglés English - Spanish. México: Ediciones Larousse. Primera edición- $3^{\mathrm{a}}$ reimpresión.

Lázaro Carreter, Fernando. [1953 (1987)]. Diccionario de términos filológicos. Madrid: Editorial Gredos.

Leeandowski, Theodor. (1992). Diccionario de lingüística. Madrid: Ediciones Cátedra.

Mahecha Mahecha, Viviana y Pedraza Pedraza, María Betulia. (2010). El diccionario escolar y algunas de las problemáticas que surgen en su elaboración. Colombia: Universidad Pedagógica Nacional. Folios no. 29.

Maldonado González, Concepción. (1993). ¿Cómo elegir un diccionario escolar?. Alacena, 17, 1993, págs. 12-13. Cuadernos de Lengua Española, Madrid: Arco/ Libros, 1997 (en prensa).

Maldonado González, Concepción. (1998). El uso del diccionario en el aula. Madrid: Arco Libros.

Martín G., Josefa. (1999) El diccionario en la enseñanza del español. Madrid: Arcos/ Libros.
Martín, A. D. (2009). El diccionario en el aula. Innovación y experiencias, 1-8.

Martínez de Sousa. (1995) Diccionario de lexicografía práctica. Barcelona: Vox.

Ministerio de Educación Pública y Cultura (2005). Programa de Estudios. Español. I y II Ciclo de la Educación General Básica. San José: Departamento de publicaciones, ministerio de Educación Pública.

Morales, Amparo. (sf). Diccionario práctico del estudiante. Organización APLE. pp. 1-4.

Murillo, Marielos. (2011). Creciendo en palabras. La enseñanza del vocabulario en la escuela primaria. Manual para maestros de primer ciclo de la educación general básica/. Costa Rica: Editorial de la Universidad de Costa Rica.

Murillo, Marielos. (2008). La educación lingüística en la escuela primaria costarricense. Kañina, Rev. Artes y letras, Univ. Costa Rica. Vol. XXXI, 69 - 91.

Navarro, Clara. (2004). Los distintos tipos de diccionarios. (sin editorial).

Océano. (1993). Diccionario conciso. Sinónimos y antónimos. España: Editorial Océano.

Océano. (1994). Diccionario enciclopédico ilustrado. España: Editorial Océano.

Palacios García, Isabel María. (2009). "El diccionario: aplicaciones prácticas en el aula". Granada, España.

Pérez, M. (2010). El primer diccionario escolar. (no hay más datos)

Prado Aragonés, Josefina. (2004). Didáctica de la lengua y la literatura para educar en el Siglo XXI. España: Editorial La Muralla. 
Prado Aragonés, Josefina. (2000). "El Diccionario y la enseñanza de la lengua". En Rusteler y Prado Aragonés (Editores). Tendencias en el diccionario como objeto de estudio lingüístico y didáctico. España: Universidad de Huelva. 171-191pp.

Prado Aragonés, Josefina. (2005). "El uso del diccionario para la enseñanza de la lengua: consideraciones metodológicas". Kañina, Rev. Artes y letras, Univ. Costa Rica. Vol. XXIX, Pág. $19-28$

Prado Aragonés, Josefina. (1996). Tu diccionario. Descúbrelo y aprende a manejarlo. Huelva: Delegación Provincial de la consejería de Educación y ciencia de Huelva.

Prado Aragonés, Josefina. (2005). Estrategias y actividades para el uso del diccionario en el aula. En Rusteler y Prado Aragonés (Editores). Tendencias en el diccionario como objeto de estudio lingüístico y didáctico. España: Universidad de Huelva.

Ramón Sopena. Editores (1981). ARISTOS. Diccionario ilustrado de la lengua española. Barcelona: Editorial Ramón Sopena.

Ríos González, Gabriela. (2010) “Taller para el uso del diccionario en el aula". Kañina, Rev. Artes y letras, Univ. Costa Rica. Vol. XXXIV (especial), Pág. 161 - 172.

Ríos González, Gabriela. (2001). “La lexicografía en la internet”. Costa Rica. Kañina, Rev.
Artes y Letras, Univ. Costa Rica. Vol. XXV, Pág. $31-35$

Rodríguez Gil, Ana María. (2006). Reflexiones sobre el uso del diccionario en la clase de ELE. Congreso nacional de ANPE. Noruega.

Rodríguez, A. (2006). Reflexiones sobre el uso del diccionario en la clase. Noruega.

Sánchez Corrales, Víctor. (1988). "Lexicografía del español en Costa Rica, Visión crítica". Kañina: Rev. Artes y Letras, Univ. Costa Rica. Vol. XIV, 147-156.

Sánchez Corrales, Víctor. (1996). "La tarea lexicográfica y proyectos de investigación”. Kañina,. Vol. XX (2), 111 $-117$.

Sánchez Muñoz, Trinidad. (s.f.). "Los diccionarios escolares a comienzos del siglo XXI". España: Centro de investigaciones lingüísticas de la Universidad de Salamanca.

Strauss Anselm. (2002). Bases de la Investigación Cualitativa. Universidad de Antioquía. Sage Publications. Colombia.

Van Patten de Ocampo, Elía María. (1993). Elementos de la bibliotecología para la biblioteca escolar y centros de recursos para el aprendizaje. Costa Rica: .EUNED. 
